\title{
Frontal Fibrosing Alopecia and Vitiligo: Coexistence or True Association?
}

\author{
Alexandros C. Katoulis ${ }^{a}$ Konstantina Diamanti ${ }^{a}$ Dimitrios Sgouros ${ }^{a}$ \\ Aikaterini I. Liakou ${ }^{a}$ Antigoni Alevizou ${ }^{a} \quad E^{2}$ angelia Bozi ${ }^{a}$ Vasileia Damaskou $^{b}$ \\ loannis Panayiotides ${ }^{\mathrm{b}}$ Dimitrios Rigopoulos ${ }^{\mathrm{a}}$
}

a 2 nd Department of Dermatology and Venereology, and ${ }^{\mathrm{b}}$ 2nd Department of Pathology, National and Kapodistrian University of Athens Medical School, "Attikon" General University Hospital, Athens, Greece

\section{Established Facts}

- Frontal fibrosing alopecia (FFA) is a non-uncommon lymphocyte-mediated cicatricial alopecia.

- Immunologic and hormonal factors have been implicated in its pathogenesis.

\section{Novel Insights}

- A considerable prevalence of vitiligo was observed in our series of patients diagnosed with FFA.

- Potential immunologic mechanisms interrelating these two dermatoses are presented, suggesting that this association may be more than coincidental.

\section{Keywords}

Alopecia $\cdot$ Vitiligo $\cdot$ Frontal fibrosing alopecia $\cdot$ Coexistence

\section{Abstract}

Frontal fibrosing alopecia (FFA) is a primary lymphocytic cicatricial alopecia characterized by a progressive band-like recession of the frontotemporal hairline and frequent loss of the eyebrows. It predominantly affects postmenopausal women. Coexistence of FFA and vitiligo is rarely reported in the literature. We retrospectively studied 20 cases diagnosed with FFA in a 14-month period in our Department. Among them, there were 2 cases, a 72-year-old woman and a 48-year-old man, who developed FFA on preexisting vitili- go of the forehead. Anatomical colocalization of the two dermatoses supports the notion that a causal link may exist and their association may not be coincidental. We suggest that interrelated immunologic events and pathologic processes may underlie both these skin conditions.

(C) 2016 S. Karger AG, Basel

\section{Introduction}

Frontal fibrosing alopecia (FFA) is a primary lymphocytic cicatricial alopecia characterized by a progressive symmetric recession of the frontotemporal hairline, frequent loss of the eyebrows, and, less often, alopecia of the

\section{KARGER}

E-Mail karger@karger.com www.karger.com/sad
(C) 2016 S. Karger AG, Basel

2296-9195/16/0024-0152\$39.50/0
Prof. Alexandros C. Katoulis

"Attikon" General University Hospital

Rimini Street 1, Chaidari

GR-12462 Athens (Greece)

E-Mail alexanderkatoulis@yahoo.co.uk 
Fig. 1. a, b Coexistence of vitiligo of the face with FFA of the frontal hairline and the eyebrows in a 72-year-old woman.
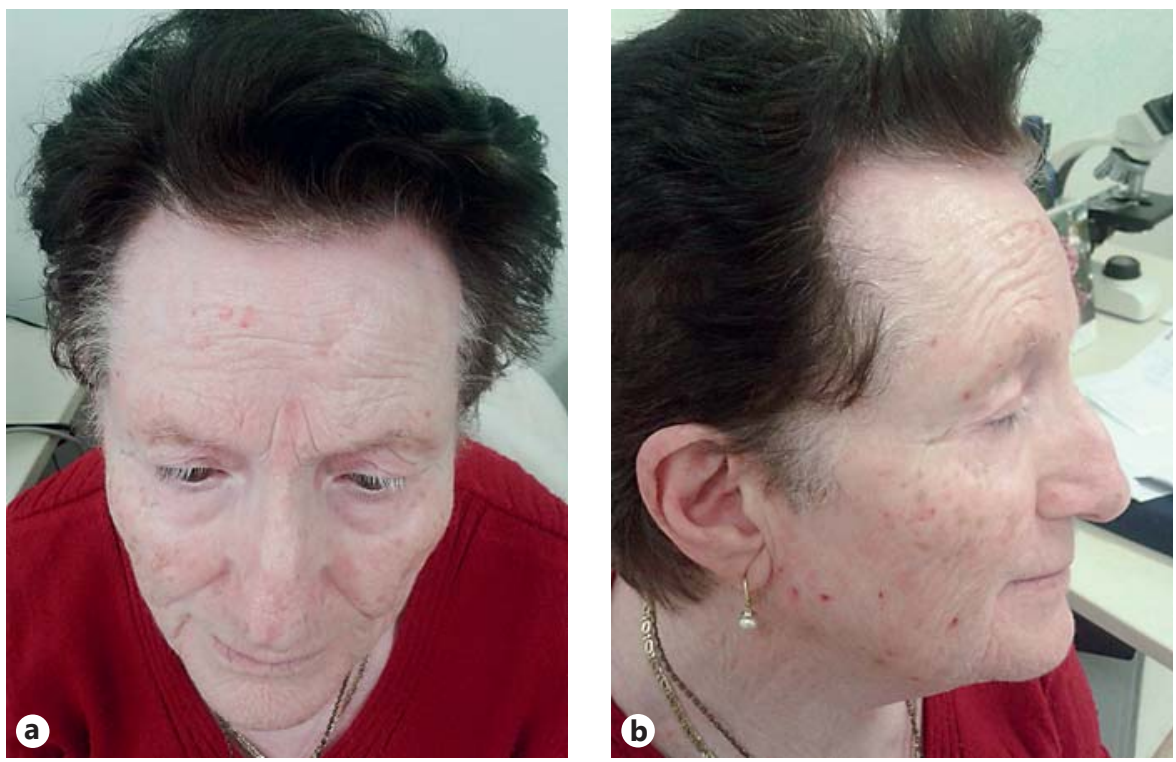

axillae, the pubic area, or the limbs [1]. It is almost exclusively seen in postmenopausal women $[1,2]$. Its occurrence in men is rare, with only 25 cases reported to date [3-14].

The etiopathogenesis of FFA remains obscure, yet a key element seems to be the destruction of the epithelial hair follicle stem cells located in the bulge region by a lymphocytic infiltrate [15]. In addition, the localization at the frontal hairline, the predominance of postmenopausal women, the increased incidence of early menopause [11], and the clinical improvement with anti-androgen drugs [11, 16-18] suggest that hormonal influences may also play an important role. The true nature of FFA is yet debated. It is not clear if it represents a distinct type of lymphocyte-mediated cicatricial alopecia or a variant of lichen planopilaris (LPP) with selective topography $[19,20]$.

In the literature, there are rare reports of vitiligo coexisting with FFA. Herein, we present two additional cases of FFA that developed on preexisting vitiligo of the forehead, and we discuss possible causal immunologic associations.

\section{Case Presentation}

We conducted a retrospective study of all histologically proven FFA cases diagnosed at the Hair Clinic of the 2nd Academic Department of Dermatology and Venereology, "Attikon" General University Hospital, Athens, Greece, from January 1, 2015 to February 29, 2016. During the period studied, 20 cases of FFA were diagnosed. Of these, $19(95 \%)$ were females with a mean age of 58 years (range 23-78) and 1 (5\%) was male, aged 48 years. Associated vitiligo was present in $2(10 \%)$ of the 20 patients included.

\section{Case 1}

A 72-year-old Caucasian presented with a 5-year history of progressive hair loss on the frontal scalp following a stressful event, accompanied by pruritus (Fig. 1a, b). Clinical examination revealed also thinning of the eyebrows, almost complete alopecia of the limbs and the pubic area, and partial loss of the axillary hair, dating back 10 years. Dermoscopic examination of the frontal hairline and eyebrows showed loss of follicular openings, mild perifollicular erythema, white scar-like depigmentation, and red dots (Fig. 2a, b). Dermoscopy-guided 3-mm punch biopsy of the scalp confirmed the diagnosis of FFA. The patient had been diagnosed with vitiligo at the age of 8 years, and at the time of the examination, she presented with generalized vitiligo. She mentioned that her mother, grandmother (father's side), brother, and sister also had vitiligo and that her daughter suffered from multiple sclerosis. No family history of FFA was given. Autoimmune thyroid disease and histologically confirmed morphea were also present in the patient, the latter of 1-year duration.

\section{Case 2}

A 48-year-old Caucasian male was referred to us with an asymptomatic recession of the frontotemporal hairline dating back 1 year. The patient had been diagnosed with vitiligo 7 years before. He had a history of severe head injury following a car accident. There was no history of FFA or autoimmune disease in his family. No patches of vitiligo were observed elsewhere. On clinical examination, a white, band-like patch on the temporal and forehead areas was present, extending below the lower margin of cicatricial skin, clinically consistent with vitiligo. Dermoscopy of the hairline showed loss of follicular orifices, lonely hair, and mild perifollicular erythema and scaling. Histological findings from the frontal hairline were consistent with FFA. 

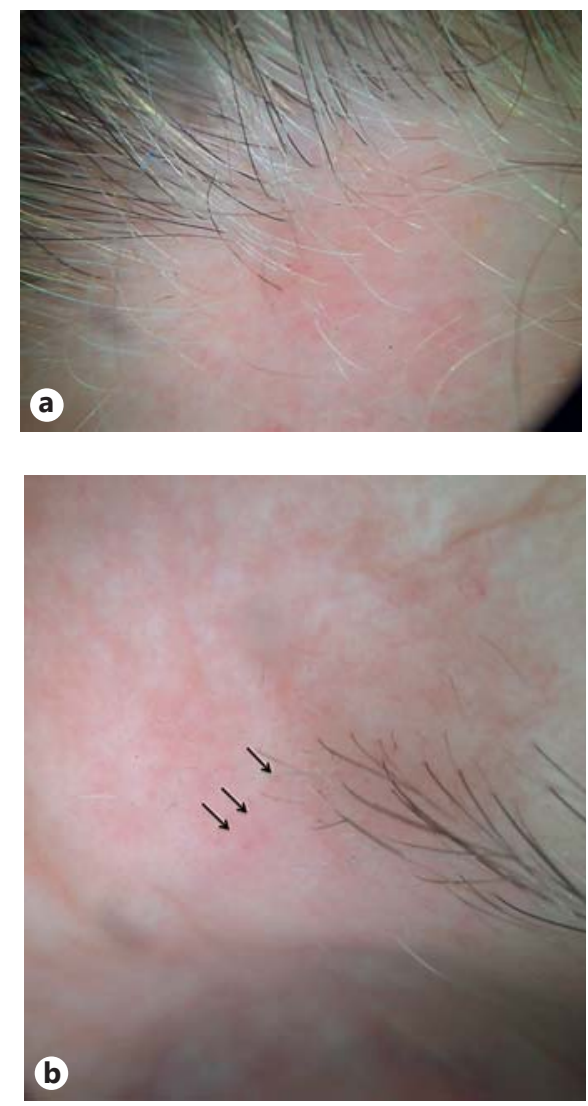

Fig. 2. a Dermoscopy of the frontal hairline showing lonely hairs and perifollicular erythema on a whitish background. b Typical red dots on an alopecic area of the left eyebrow.

\section{Discussion}

Association of FFA with vitiligo is scarcely described in the literature, with only 8 cases reported so far, to the best of our knowledge $[11,13,21,22]$. In our series, we found 2 cases of FFA coexisting with vitiligo among 20 cases included in the study. In addition, the anatomical colocalization of these two dermatoses in both of our cases may suggest that a true association may exist, and vitiligo and FFA may share common pathogenic pathways.

Lichen planus (LP) has been previously associated with vitiligo [23-31]. Furthermore, some authorities consider FFA as a variant of LPP $[19,20]$. There is evidence that most lymphocytic clones involved in both LP and vitiligo are of CD8+ cytotoxic origin [32-34]. In LP, the cytotoxic lymphocytes, alleged to target yet unknown antigen(s), conjugate to the basal layer keratinocytes and induce apoptosis of the latter. It is known that melanocytes and keratinocytes form functional units; therefore, apoptosis of keratinocytes can result in decreased melanocytic survival and apoptosis, as occurs in vitiligo. The aforementioned findings could imply that the keratinocytes of the outer root sheath, being continuous with the epidermal keratinocytes, can also express the adhesion molecules necessary for attachment of the cytotoxic lymphocytes to the affected follicles [21]. In this context, vitiligo and LPP/FFA may develop simultaneously. In addition, vitiligo could alter the exposure of dermoepidermal junction antigens identified by effector T cells of FFA, or alternatively, vitiligo may nonspecifically inactivate suppressive mechanisms on the effector cells responsible for FFA [30].

The Koebner phenomenon, which LP is well known to exhibit, could also explain the anatomical colocalization of these two dermatoses, as observed in our two cases (especially case 2 , who showed features of vitiligo solely on the frontal area). Additionally, there are several reports describing lesions of LP that developed mainly over sunexposed vitiliginous skin [25, 26, 29-31]. Extrapolating this to FFA, actinic damage in vitiliginous skin may trigger initiation of FFA on vitiligo-affected skin. However, the literature also contains cases of associated LP and vitiligo that are not readily accommodated by this theory $[24,28,35,36]$.

FFA is extremely uncommon in men. Therefore, case 2 is interesting not only because it involves a male patient, but also because, to the best of our knowledge, it is the first description of coexistence of vitiligo and FFA in a male. Another interesting point is the occurrence of FFA on the sole vitiliginous patch of the patient.

In conclusion, we report 2 cases of FFA coexisting with vitiligo in a series of 20 FFA patients studied. The anatomical colocalization supports the notion that the association is not coincidental. Both diseases have a common pathogenic background and we assume that this association may be explained by co-stimulation of immunological mechanisms, or alternatively, inactivation of nonspecific suppressor mechanisms. Koebner phenomenon related to subclinical photodamage inducing FFA preferentially over vitiliginous skin could also be considered. At present, there is insufficient evidence to resolve the uncertainties, but the possible immunologic association between FFA and vitiligo seems to merit further study.

\section{Statement of Ethics}

The two patients presented in this paper gave their consent for the above presentation. 


\section{Disclosure Statement}

There is no conflict of interest concerning this paper.

\section{References}

1 Kossard S: Postmenopausal frontal fibrosing alopecia. Scarring alopecia in a pattern distribution. Arch Dermatol 1994;130:770-774.

2 Moreno-Ramírez D, Ferrandiz L, Camacho FM: Diagnostic and therapeutic assessment of frontal fibrosing alopecia. Actas Dermosifiliogr 2007;98:594-602.

3 Stockmeier M, Kunte C, Sander CA, Wolff H: Kossard frontal fibrosing alopecia in a man. Hautarzt 2002;53:409-411.

4 Kossard S, Shiell RC: Frontal fibrosing alopecia developing after hair transplantation for androgenetic alopecia. Int J Dermatol 2005; 44:321-323.

5 Nusbaum BP, Nusbaum AG: Frontal fibrosing alopecia in a man: results of follicular unit test grafting. Dermatol Surg 2010;36:959-962.

6 Ramaswamy P, Mendese G, Goldberg LJ: Scarring alopecia of the sideburns: a unique presentation of frontal fibrosing alopecia in men. Arch Dermatol 2012;148:1095-1096.

7 Chen W, Kigitsidou E, Prucha H, Ring J, Andres C: Male frontal fibrosing alopecia with generalised hair loss. Australas J Dermatol 2014;55:e37-e39.

8 Dlova NC, Goh CL: Frontal fibrosing alopecia in an African man. Int J Dermatol 2015;54: 81-83.

9 Khan S, Fenton DA, Stefanato CM: Frontal fibrosing alopecia and lupus overlap in a man: guilt by association? Int J Trichology 2013;5: 217-219.

10 Samrao A, Chew AL, Price V: Frontal fibrosing alopecia: a clinical review of 36 patients. Br J Dermatol 2010;163:1296-1300.

11 Vañó-Galván S, Molina-Ruiz AM, SerranoFalcón C, Arias-Santiago S, Rodrigues-Barata AR, Garnacho-Saucedo G, Martorell-Calatayud A, Fernández-Crehuet P, Grimalt R, Aranegui B, Grillo E, Diaz-Ley B, Salido R, Pérez-Gala S, Serrano S, Moreno JC, Jaén P, Camacho FM: Frontal fibrosing alopecia: a multicenter review of 355 patients. J Am Acad Dermatol 2014;70:670-678.

12 Dlova NC, Jordaan HF, Skenjane A, Khoza N, Tosti A: Frontal fibrosing alopecia: a clinical review of 20 black patients from South Africa. Br J Dermatol 2013;169:939-941.

13 Banka N, Mubki T, Bunagan MJ, McElwee K, Shapiro J: Frontal fibrosing alopecia: a retrospective clinical review of 62 patients with treatment outcome and long-term follow-up. Int J Dermatol 2014;53:1324-1330.

14 AlGaadi S, Miteva M, Tosti A: Frontal fibrosing alopecia in a male presenting with sideburn loss. Int J Trichology 2015;7:72-73.

15 Vaisse V, Matard B, Assouly P, Jouannique C, Reygagne P: Postmenopausal frontal fibrosing alopecia: 20 cases. Ann Dermatol Venereol 2003;130:607-610.

16 Tosti A, Piraccini BM, Iorizzo M, Misciali C: Frontal fibrosing alopecia in postmenopausal women. J Am Acad Dermatol 2005;52:55-60.

17 Georgala S, Katoulis AC, Befon A, Danopoulou I, Georgala C: Treatment of postmenopausal frontal fibrosing alopecia with oral dutasteride. J Am Acad Dermatol 2009;61: 157-158.

18 Ladizinski B, Bazakas A, Selim MA, Olsen EA: Frontal fibrosing alopecia: a retrospective review of 19 patients seen at Duke University. J Am Acad Dermatol 2013;68:749-755.

19 Kossard S, Lee MS, Wilkinson B: Postmenopausal frontal fibrosing alopecia: a frontal variant of lichen planopilaris. J Am Acad Dermatol 1997;36:59-66.

20 Poblet E, Jiménez F, Pascual A, Piqué E: Frontal fibrosing alopecia versus lichen planopilaris: a clinicopathological study. Int J Dermatol 2006;45:375-380.

21 Miteva M, Aber C, Torres F, Tosti A: Frontal fibrosing alopecia occurring on scalp vitiligo: report of four cases. Br J Dermatol 2011;165: 445-447.

22 Tan KT, Messenger AG: Frontal fibrosing alopecia: clinical presentations and prognosis. Br J Dermatol 2009;160:75-79.

23 Gül U, Soylu S, Demiriz M: Colocalization of lichen planus and vitiligo associated with selective IgA deficiency. Skinmed 2007;6:202-203.

24 Göktay F, Mansur AT, Aydingöz IE: Colocalization of vitiligo and lichen planus on scrotal skin: a finding contrary to the actinic damage theory. Dermatology 2006;212:390-392.

25 Ujiie H, Sawamura D, Shimizu H: Development of lichen planus and psoriasis on lesions of vitiligo vulgaris. Clin Exp Dermatol 2006; 31:375-377.

26 Baghestani S, Moosavi A, Eftekhari T: Familial colocalization of lichen planus and vitiligo on sun exposed areas. Ann Dermatol 2013;25: 223-225.

27 Golchai J, Ramezanpour A: Report of a new case with four skin diseases. Dermatol Online J 2003;9:15.

28 Porter SR, Scully C, Eveson JW: Coexistence of lichen planus and vitiligo is coincidental. Clin Exp Dermatol 1994;19:366.

29 Veitch D, Kravvas G, Hughes S, Bunker C: A rare colocalization of lichen planus and vitiligo. Case Rep Dermatol Med 2015;2015: 840193.

30 Anstey A, Marks R: Colocalization of lichen planus and vitiligo. Br J Dermatol 1993;128: 103-104.

31 Sardana K, Sharma RC, Koranne RV, Mahajan S: An interesting case of colocalization of segmental lichen planus and vitiligo in a 14-year-old boy. Int J Dermatol 2002;41:508509.

32 Piguet V, Breathnach SM, Le Cleach L: Lichen planus and lichenoid disorders; in Griffiths $\mathrm{C}$, Barker J, Bleiker T, Chalmers R, Creamer D (eds): Rook's Textbook of Dermatology, 9th edition. West Sussex, Wiley-Blackwell, 2016, pp 37.1-37.20.

33 Glassman SJ: Vitiligo, reactive oxygen species and T-cells. Clin Sci (Lond) 2011;120:99-120.

34 Farhi D, Dupin N: Pathophysiology, etiologic factors, and clinical management of oral lichen planus, part I: facts and controversies. Clin Dermatol 2010;28:100-108.

35 Wayte J, Wilkinson JD: Unilateral lichen planus, sparing vitiliginous skin. Br J Dermato 1995; 133:817-818.

36 Baran R, Ortonne JP, Perrin C: Vitiligo associated with a lichen planus border. Dermatology 1997;194:199. 